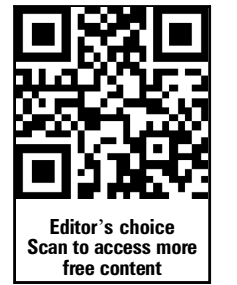

- Additional material is published online only. To view please visit the journal online (http://dx.doi.org/10.1136/ gutjnl-2012-303261).

For numbered affiliations see end of article.

Correspondence to Dr Snorri S Thorgeirsson, Center of Excellence in Integrative Cancer Biology and Genomics, Laboratory of Experimental Carcinogenesis, Center for Cancer Research, National Cancer Institute, $\mathrm{NIH}_{\text {, }}$ 37 Convent Drive MSC 4262 Building 37, Room 4146A, Bethesda, MD 20892-4262, USA;

snorri thorgeirsson@nih.gov; Dr Itzhak Avital,

Bon Secours Cancer Institute, 6605 West Broad Street, Richmond, VA 23230, USA itzhak.avital@gmail.com

H-WX and CMA contributed equally.

Received 8 July 2012 Revised 21 January 2013 Accepted 22 January 2013 Published Online First 14 February 2013

\section{Linked}

- http://dx.doi.org/10.1136/ gutjnl-2013-304564

To cite: Xin H-W, Ambe CM, Hari DM, et al. Gut

2013;62:1777-1786

\title{
Label-retaining liver cancer cells are relatively resistant to sorafenib
}

\author{
Hong-Wu Xin, ${ }^{1}$ Chenwi M Ambe, ${ }^{1}$ Danielle M Hari, ${ }^{1}$ Gordon W Wiegand, ${ }^{1}$ Tyler C Miller, ${ }^{1}$ \\ Jin-Qiu Chen, ${ }^{2}$ Andrew J Anderson, ${ }^{1}$ Satyajit Ray, ${ }^{1}$ John E Mullinax, ${ }^{1}$ Tomotake Koizumi, ${ }^{1}$ \\ Russell C Langan, ${ }^{1}$ Douglas Burka, ${ }^{1}$ Michelle A Herrmann, ${ }^{2}$ Paul K Goldsmith, ${ }^{2}$ \\ Alexander Stojadinovic, ${ }^{3,4}$ Udo Rudloff, ${ }^{1}$ Snorri S Thorgeirsson, ${ }^{5}$ Itzhak Avital ${ }^{1,4,6}$
}

\section{ABSTRACT \\ Objective The standard therapy for advanced} hepatocellular carcinoma (HCC) is sorafenib, with most patients experiencing disease progression within 6 months. Label-retaining cancer cells (LRCC) represent a novel subpopulation of cancer stem cells (CSC). The objective was to test whether LRCC are resistant to sorafenib.

Methods We tested human HCC derived LRCC and non-LRCC before and after treatment with sorafenib. Results LRCC derived from human HCC are relatively resistant to sorafenib. The proportion of $L R C C$ in $\mathrm{HCC}$ cell lines is increased after sorafenib while the general population of cancer cells undergoes growth suppression. We show that LRCC demonstrate improved viability and toxicity profiles, and reduced apoptosis, over non-LRCC. We show that after treatment with sorafenib, LRCC upregulate the CSC marker aldehyde dehydrogenase 1 family, wingless-type MMTVintegration-site family, cell survival and proliferation genes, and downregulate apoptosis, cell cycle arrest, cell adhesion and stem cells differentiation genes. This phenomenon was accompanied by non-uniform activation of specific isoforms of the sorafenib target proteins extracellular-signal-regulated kinases and v-aktmurine-thymoma-viral-oncogene homologue (AKT) in LRCC but not in non-LRCC. A molecular pathway map for sorafenib treated LRCC is proposed.

Conclusions Our results suggest that HCC derived LRCC are relatively resistant to sorafenib. Since LRCC can generate tumours with as few as 10 cells, our data suggest a potential role for these cells in disease recurrence. Further investigation of this phenomenon might provide novel insights into cancer biology, cancer recurrence and drug resistance with important implications for the development of novel cancer therapies based on targeting LRCC.

\section{INTRODUCTION}

Hepatocellular carcinoma (HCC) represents the third most common cause of cancer death. ${ }^{1}$ The SHARP (Sorafenib HCC assessment randomized protocol) trial established the small molecule tyrosine kinase inhibitor (TKI), sorafenib, as the standard of care for patients with advanced HCC. ${ }^{2}$ The median time to progression improved from 2.8 to 5.5 months in patients with advanced HCC treated with sorafenib. While sorafenib is the first systemic agent to show improvement in overall survival for patients with

\section{Significance of this study}

What is already known on this subject?

- Response rate to sorafenib in patients with advanced hepatocellular carcinoma (HCC) is approximately $2 \%$ with most patients' progress within 6 months after treatment with sorafenib.

- Cancers are composed of stem-like cancer cells and non-stem cancer cells. It has been hypothesised that stem-like cancer cells are resistant to therapy.

- Recently, label-retaining cancer cells (LRCC) were described as novel and potent cancer stem cells. It is not known whether any HCC derived cancer stem cells or LRCC are resistant to sorafenib.

\section{What are the new findings?}

- This is the first report showing that LRCC, a recently described novel class of HCC derived stem-like cancer cells, are resistant to the targeted therapy sorafenib.

- Resistance to sorafenib is manifested by improved viability, toxicity profiles and reduced apoptosis over non-LRCC. This phenomenon was accompanied by stem cell-like gene expression profile, and activation of specific isoforms of the sorafenib target proteins extracellular-signal-regulated kinases and v-akt-murine-thymoma-viral-oncogenehomologue.

How might it impact on clinical practice in the foreseeable future?

- These data suggest a role for LRCC in HCC recurrence. Further understanding of sorafenib-resistant LRCC may facilitate the development of novel cancer therapeutic drugs that target specifically cancer stem cell/LRCC or their mechanism to resist therapy, leading to a better cancer control.

HCC, the improvement in outcomes is modest. Most patients will suffer disease recurrence and die. The basis for HCC recrudescence after treatment with sorafenib is unknown.

Sorafenib is an oral TKI. ${ }^{3}$ It has activity against receptor tyrosine kinases (VEGFR-1, 2 and 3, 
PDGFR- $\beta$, c-Kit, Fms-related tyrosine kinase (FLT) 3 and ret proto-oncogene; table 1) and cytoplasmic kinases (Raf-1, B-Raf, C-Raf; table 1). ${ }^{3}$ Sorafenib has antiangiogenic, antiproliferative and pro-apoptotic effects. ${ }^{3}$ Sorafenib indirectly affects multiple target genes; 60 key genes were reported in the literature and are herein referred to as sorafenib target genes (STG; table 1). The question is, 'Why is it that a targeted agent such as sorafenib that has effects on multiple receptors, pathways and genes, has only a short-lived, clinically meaningful treatment effect, lasting on average only a few months' ? ${ }^{3}$

Recent data suggest that cancers contain cells with stem-like characteristics, that is, cancer stem cells (CSC; also referred to as tumour-initiating cells or TIC). ${ }^{4} 5$ It has been hypothesised that CSC are responsible for therapeutic failure. Based on this hypothesis, current therapies for advanced cancers effectively target the bulk of the tumour but less effectively target the CSC. The result is a temporarily reduction in the tumour mass only to reoccur in a short time. Evidence supporting this hypothesis suggested that some CSC are resistant to anticancer drugs based on increased expression of antiapoptotic proteins and/or ATP-binding-cassette transporters. ${ }^{5} 6$ This hypothesis continues to be controversial. Moreover, there is a paucity of data suggesting that CSC are indeed resistant to targeted therapy. ${ }^{67}$

Label-retaining cells (LRC) are associated with populations of cells enriched with adult tissue stem cells. ${ }^{8} 9$ Solid organ cancers, like liver cancer, develop in tissues found to harbour LRC. ${ }^{9}$ Recently, we demonstrated that label-retaining cancer cells (LRCC) undergo asymmetric cell division, and represent a unique subpopulation of tumour-initiating stem-like cells with pluripotency gene expression profile, defining LRCC as a novel subpopulation of CSC. ${ }^{10}{ }^{11}$ It is unknown whether LRCC are resistant to targeted therapies.

We undertook this study to test whether HCC derived LRCC are resistant to sorafenib. Here we show that LRCC are

Table 1 Evidence based (published) sorafenib target genes

\begin{tabular}{|c|c|c|c|}
\hline Symbol & Gene name & Location & Function \\
\hline AIFM1 & Apoptosis-inducing factor, mitochondrion-associated, 1 & Cytoplasm & Apoptosis \\
\hline Akt1 & V-akt murine thymoma viral oncogene homologue & Cytoplasm & Proliferation, survival \\
\hline ALDH1A1 & Aldehyde dehydrogenase 1 family, member $\mathrm{A} 1$ & Cytoplasm & Alcohol metabolism, cancer stem cells marker \\
\hline ATG5 & ATG5 autophagy-related 5 homologue & Cytoplasm & Autophagy, apoptosis \\
\hline BAD & BCL2-associated agonist of cell death & Cytoplasm & Apoptosis \\
\hline BAK1 & BCL2-antagonist/killer 1 & Cytoplasm & Apoptosis \\
\hline BAX & BCL2-associated X protein & Cytoplasm & Apoptosis \\
\hline $\mathrm{BCL} 2 \mathrm{~L} 1$ & BCL2-like 1 & Cytoplasm & Survival \\
\hline BID & $\mathrm{BH} 3$ interacting domain death agonist & Cytoplasm & Apoptosis \\
\hline BRAF & V-raf murine sarcoma viral oncogene homologue b1 & Cytoplasm & Proliferation \\
\hline CASP3, 7, 8, 9 & Caspase $3,7,8,9$ apoptosis-related cysteine peptidase & Cytoplasm & Apoptosis \\
\hline CCND 1, 3 & Cyclin D1, 3 & Nucleus & Cell cycle \\
\hline CDK 4, 6 & Cyclin-dependent kinase 4, 6 & Nucleus & Cell cycle \\
\hline CFLAR & CASP8 and FADD-like apoptosis regulator & Cytoplasm & Survival \\
\hline CRKL & V-crk sarcoma virus CT10 oncogene homologue & Cytoplasm & Proliferation \\
\hline CYP2B6, C19, C8, D6 & Cytochrome P450, family 2 & Cytoplasm & Drug metabolism, lipid synthesis \\
\hline CYP3A4 & Cytochrome P450, family 3, subfamily A, polypeptide 4 & Cytoplasm & Drug metabolism, lipid synthesis \\
\hline DDIT3 & DNA-damage-inducible transcript 3 & Nucleus & Adipogenesis, erythropoiesis, apoptosis \\
\hline DIABLO & Diablo, IAP-binding mitochondrial protein & Cytoplasm & Survival \\
\hline DOK1 & Docking protein 1, $62 \mathrm{kDa}$ (downstream of tyrosine kinase 1) & Membrane & Proliferation, survival \\
\hline EIF2A, 2AK3, 2S1, 4E, 4EBP1 & Eukaryotic translation initiation factor, $65 \mathrm{kda}$ & Cytoplasm & Protein translation, survival \\
\hline ERN1 & Endoplasmic reticulum to nucleus signalling 1 & Cytoplasm & Apoptosis, stress response \\
\hline FAS & Fas (tumor necrosis factor receptor superfamily, member 6) & Membrane & Apoptosis \\
\hline FLT 3, 4 & Fms-related tyrosine kinase 3, 4 & Membrane & Proliferation, survival \\
\hline HIF1A & Hypoxia inducible factor $1, \alpha$ subunit (basic helix-loop-helix transcription factor) & Nucleus & Apoptosis, hypoxia response \\
\hline HSPA5 & Heat shock 70 kDa protein 5 (glucose-regulated protein, 78 kDa) & Cytoplasm & Stress response \\
\hline KDR & Kinase insert domain receptor (a type III receptor tyrosine kinase) & Membrane & Proliferation, survival \\
\hline KIT & V-kit Hardy-Zuckerman 4 feline sarcoma viral oncogene homologue & Membrane & Proliferation, survival \\
\hline MAPK1, 3 & Mitogen-activated protein kinase 1,3 & Cytoplasm & Proliferation \\
\hline MCL1 & Myeloid cell leukaemia sequence 1 (BCL2-related) & Cytoplasm & Survival/apoptosis \\
\hline PDGFRB & Platelet derived growth factor receptor, $\beta$ polypeptide & Membrane & Proliferation, survival \\
\hline PPP1R15A & Protein phosphatase 1, regulatory (inhibitor) subunit $15 \mathrm{~A}$ & Cytoplasm & Apoptosis, DNA damage response \\
\hline Ptk2 & Protein tyrosine kinase & Cytoplasm & Proliferation \\
\hline RAF1 & V-raf-1 murine leukaemia viral oncogene homologue 1 & Cytoplasm & Proliferation \\
\hline RELA & V-rel reticuloendotheliosis viral oncogene homologue $A$ & Nucleus & Proliferation, survival \\
\hline RPS6KB1 & Ribosomal protein s6 kinase, 70 kda, polypeptide 1 & Cytoplasm & Proliferation, protein synthesis \\
\hline SNRPE & Small nuclear ribonucleoprotein polypeptide E & Nucleus & Transcription, RNA processing \\
\hline STAT5a/b & Signal transducer and activator of transcription $5 B$ & Cytoplasm & Proliferation, survival \\
\hline TWIST2 & Twist homologue 2 (drosophila) & Nucleus & Epithelia-mysenchymal transition \\
\hline VIM & Vimentin & Cytoplasm & Epithelia-mysenchymal transition \\
\hline
\end{tabular}


relatively resistant to sorafenib, thereby providing the first published report showing that CSC (HCC derived LRCC) are resistant to a targeted anticancer agent. Because as few as 10 LRCC are needed for tumour initiation, ${ }^{11}$ our results suggest that recurrent HCC after treatment with sorafenib potentially could be explained by the relative resistance of LRCC to sorafenib. ${ }^{11}$ Our data suggest that future therapies against HCC should also target specifically the LRCC and/or the mechanism underlying their relative resistance to sorafenib opening the doors to the development of new class of anticancer drugs.

\section{MATERIALS AND METHODS}

\section{Human liver cancer cells lines}

Human HCC cells PLC/PRF/5 and SK-Hep-1 (ATCC, Manassas, Virginia, USA, http://www.atcc.org) and $\mathrm{HuH}-7$ (Japan Health Sciences Foundation, Tokyo, Japan, http://www. jhsf.or.jp) were cultured in 44\% Dulbecco's modified Eagle's medium, 44\% Ham's F-12, 10\% fetal calf serum, 1\% glutamine and $1 \%$ ampicillin/streptomycin (Invitrogen Corp, Grand Isle, New York, USA, http://www.invitrogen.com).

\section{Growth curves}

We generated growth curves with/without clinically relevant sorafenib concentrations: PLC/PRF/5 (6.4 uM), HuH-7 (6.4 uM) and SK-Hep-1 $(4 \mathrm{uM}) .{ }^{12}$ The numbers of live cells were determined $8 \mathrm{~h}$ apart $(\mathrm{n}=3)$ using Cellometer Auto T4 (Nexcelom Bioscience, Lawrence, Massachusetts, USA, http://www.nexcelom.com). Acquired numbers were averaged and plotted (figure 1).

\section{Isolation and analysis of live LRCC and non-LRCC with or without sorafenib treatment}

Live LRCC and non-LRCC were isolated from HCC cell lines as we previously described (figure 2A,B and online supplementary table S1). ${ }^{10} 11$

\section{Viability, toxicity and apoptosis assays}

We used the ApoTox-Glo assay kit (Promega, Madison, Wisconsin, USA, http://www.promega.com) according to manufacturer's protocol. We isolated and cultured LRCC and non-LRCC from HCC cell lines in 96-well plates with or without sorafenib for eight cell cycles.

\section{MEK, ERK and AKT protein kinase analysis}

NanoPro 1000 is an automated capillary based isoelectricfocusing immunoassay system (ProteinSimple, Santa Clara, California, USA, http://www.proteinsimple.com) (table 1). Protein isolation, detection and quantification were done as per manufacturer's instructions (see online supplementary data).
Gene expression analysis: real-time qRT-PCR

Live LRCC and non-LRCC cells were isolated and RNA was extracted according to manufacturer's protocol (QIAGEN, Valencia, California, USA, http://www.qiagen.com). Real-time quantitative-reverse-transcription-PCR (qRT-PCR) for customised SuperArrays was done in triplicates following the manufacturer's protocol (SABiosciences, Frederick, MD, http://www.sabiosciences. com). Cross-threshold values were analysed using the SABioscience software (SABiosciences, Valencia, California, USA, http://www. sabiosciences.com). Regulations more than twofold were considered above the technical error threshold.

\section{Pathway analysis}

Analyses were done using Ingenuity Pathway Analysis software (IPA 9.0, Ingenuity Systems, Inc., Redwood City, California, USA, http://www.ingenuity.com).

\section{Statistics}

For comparisons between two samples, we used the two-tailed Student $\mathrm{t}$ test to test the null hypothesis that the means of the two samples are equal. Two tailed $p<0.05$ was considered statistically significant. In all figures, an asterisk was added when $p<0.05$. The error bars represent SEM for figures 1 and 2, SD for figure 3 and SEM for figures 4-6.

\section{RESULTS}

\section{The relative proportion of LRCC is significantly increased} after treatment with sorafenib

We hypothesised that if LRCC are resistant to sorafenib then the proportion of LRCC relative to non-LRCC in whole cell populations of HCC should increase after treatment with sorafenib. To test this hypothesis, we first validated the efficacy of sorafenib in our laboratory on all tested bulk HCC cell lines. We tested clinically relevant sorafenib dosages (Materials and methods section). ${ }^{12}$ Cell growth curves with or without sorafenib demonstrated that sorafenib effectively inhibited the growth of all HCC cell lines tested (figure 1).

All the cell lines were composed of LRCC and non-LRCC (see online supplementary table S1). ${ }^{10}{ }^{11}$ Because LRCC represent a unique class of TIC or CSC, ${ }^{10}{ }^{11}$ we wanted to test the effects of sorafenib on the relative proportion of LRCC in HCC. Previously, we developed a method for the isolation of live LRCC (figure 2A, Materials and methods section). ${ }^{10}{ }^{11} \mathrm{In}$ brief, liver cancer cell lines (PLC/PRF/5, HuH-7 and SK-Hep-1) were first pulsed with Cyanine-5-deoxyuridinetriphosphate (Cy5-dUTP) and empty-dUTP (negative control). After one cell cycle, Cy5 + high cells were sorted by flow cytometry and chased in culture with and without clinically relevant sorafenib
Figure 1 Sorafenib effectively inhibits cell growth of tested hepatocellular carcinoma (HCC) cell lines. Growth curves of HCC cell lines treated with and without sorafenib: $(A)$ PLC/PRF/5 ( $p=0.0041),(B) \mathrm{HuH}-7$ $(p=0.0094)$ and $(C)$ SK-Hep-1 $(p=0.0042)\left({ }^{*} p<0.05\right)$.
A

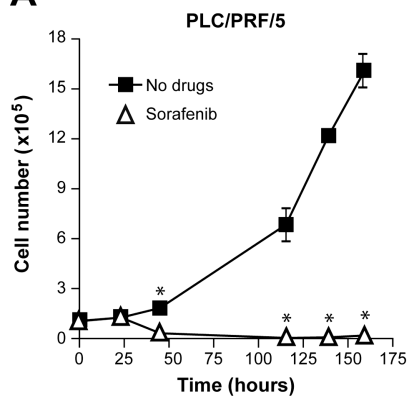

B

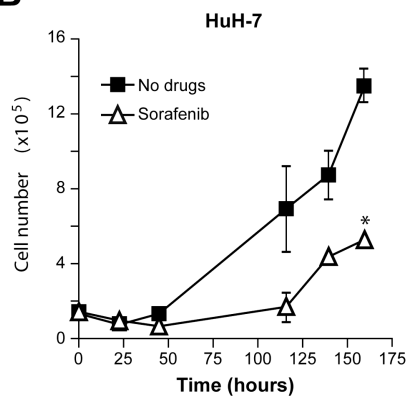

C

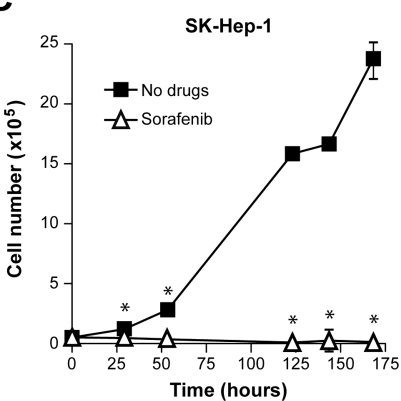


A
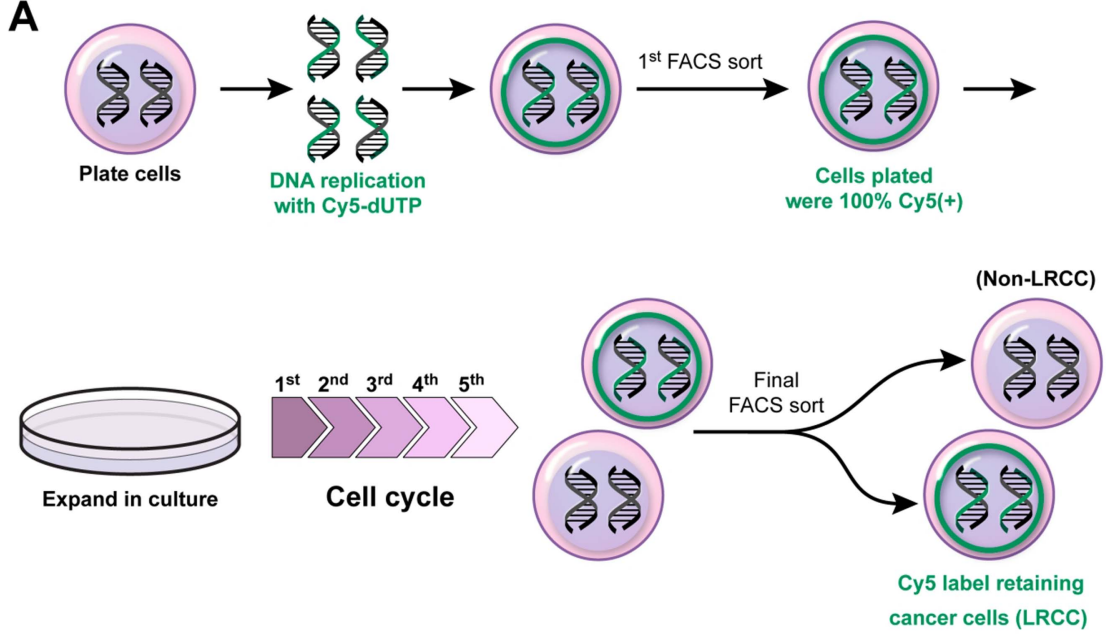

B
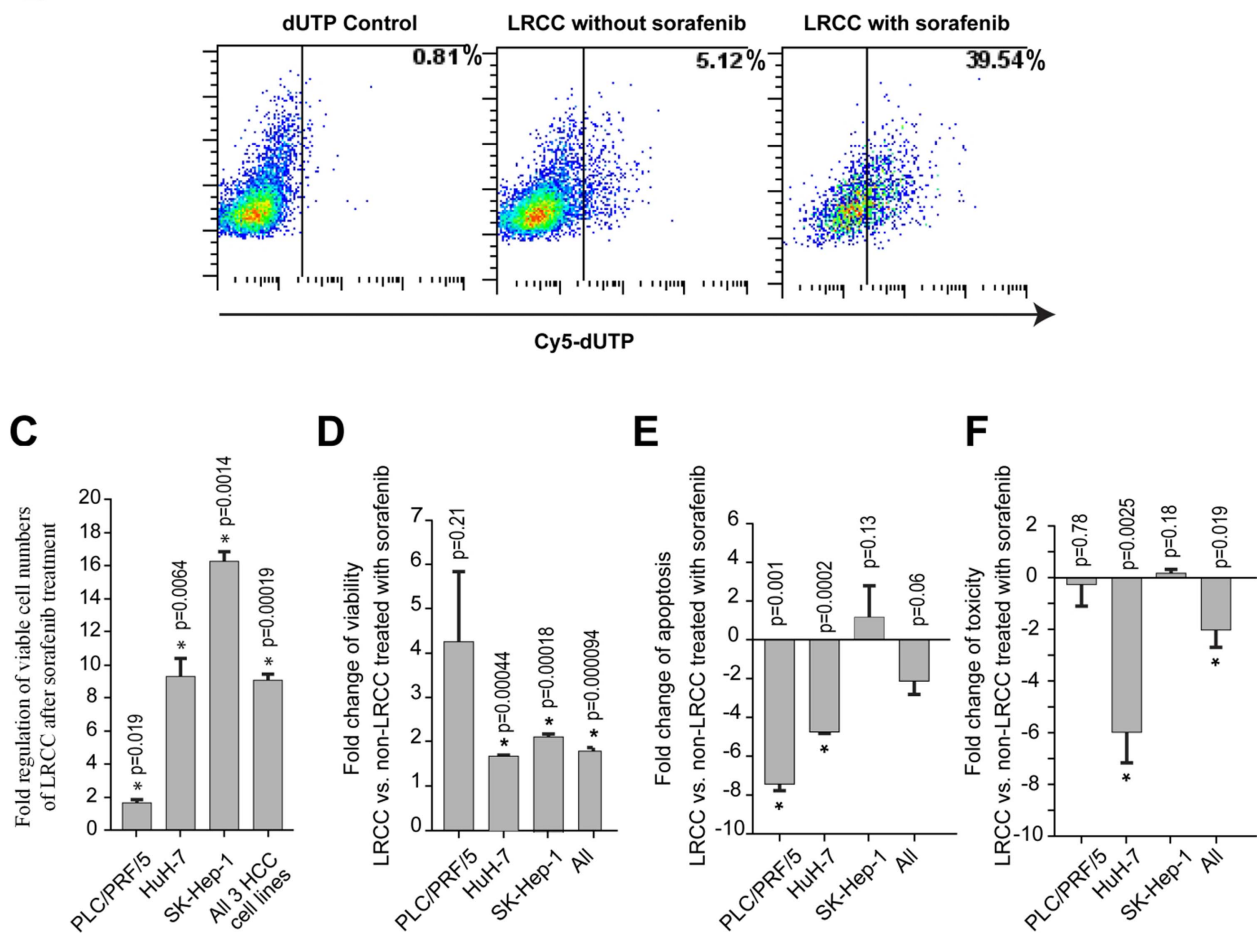

Figure 2 Label-retaining cancer cells (LRCC) are relatively resistant to sorafenib. (A) Isolation of hepatocellular carcinoma (HCC) derived LRCC. The full method is described in our previous reports. ${ }^{10}{ }^{11}$ Whole cell populations of HCC cells were labelled with Cy5-DNA-nucleotides (pulse phase). Subsequently, Cy5-positive-high cells were sorted and grown for eight cell cycles (chase phase). Finally, Cy5-positive-high cells (99\% pure) and Cy5-negative cells were sorted as LRCC and non-LRCC, respectively. (B)-(C) The relative proportion of LRCC is increased after sorafenib treatment, and data in (B) are from HuH-7. Compared with non-LRCC, LRCC exhibit better viability (D), better apoptosis profile (E) and better toxicity profile $(F)\left({ }^{*} p<0.05\right)$.

concentrations for eight cell cycles $(n=6) \cdot{ }^{10-12}$ After eight cell cycles, LRCC (Cy5+ high cells) and non-LRCC (Cy5-negative cells) were sorted by flow cytometry and tested. Here we show that after treatment of the HCC cell lines with clinically relevant dose of sorafenib, the relative proportion of LRCC is increased by $9.1 \pm 0.4$ fold, figure $2 \mathrm{~B}, \mathrm{C})$. The increased relative proportion of LRCC was not identical in all tested cell lines: PLC/PRF/5 (1.6 \pm 0.2 fold), HuH-7 $(9.3 \pm 1.1)$ and SK-Hep-1 (16.3 \pm 0.6 fold). Our data showed that the relative proportion of LRCC was increased after treatment with sorafenib suggesting that when compared with non-LRCC, LRCC are significantly more resistant to sorafenib. In figure $1 \mathrm{~A}$, we show cell numbers after treatment with sorafenib (PLC/PRF/5). Although the graph seems to be at ' 0 ', there are resistant whole population cells live in each of the time points. In figure $2 \mathrm{C}$, we show the viability of LRCC; although, overall most of the cells are inhibited by sorafenib, the LRCC are less inhibited than the non-LRCC and thus their relative proportion is increased as expected from CSC. Figure 2C shows absolute viability of LRCC in the dynamic whole population cells after treatment with sorafenib while figure 2D shows relative viability of isolated LRCC versus non-LRCC. 
A
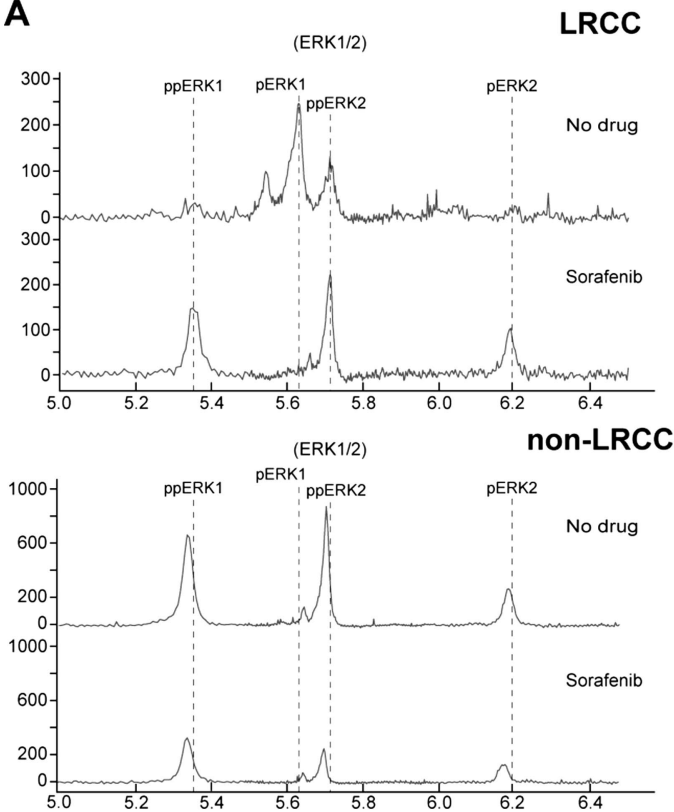

B
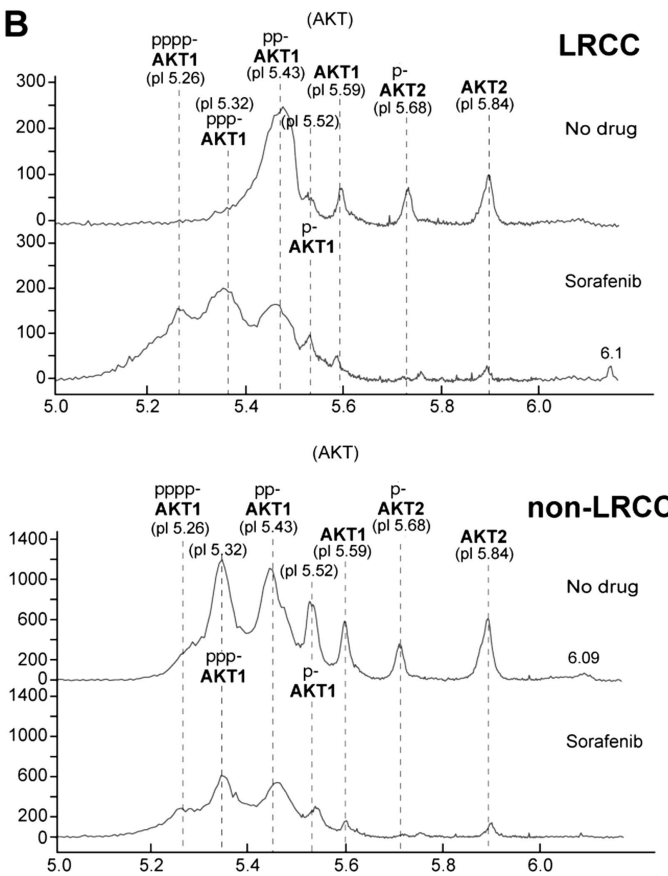
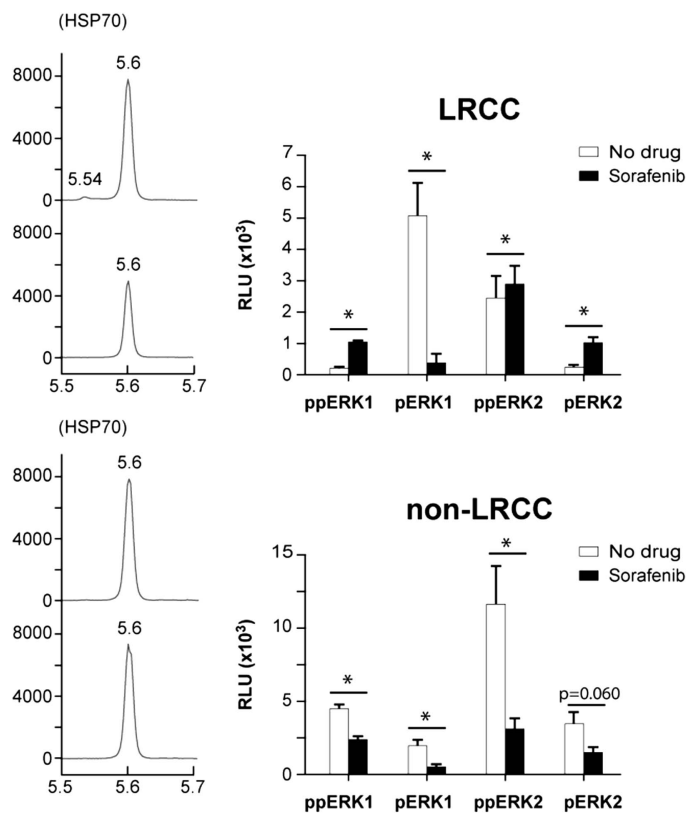

(HSP70)
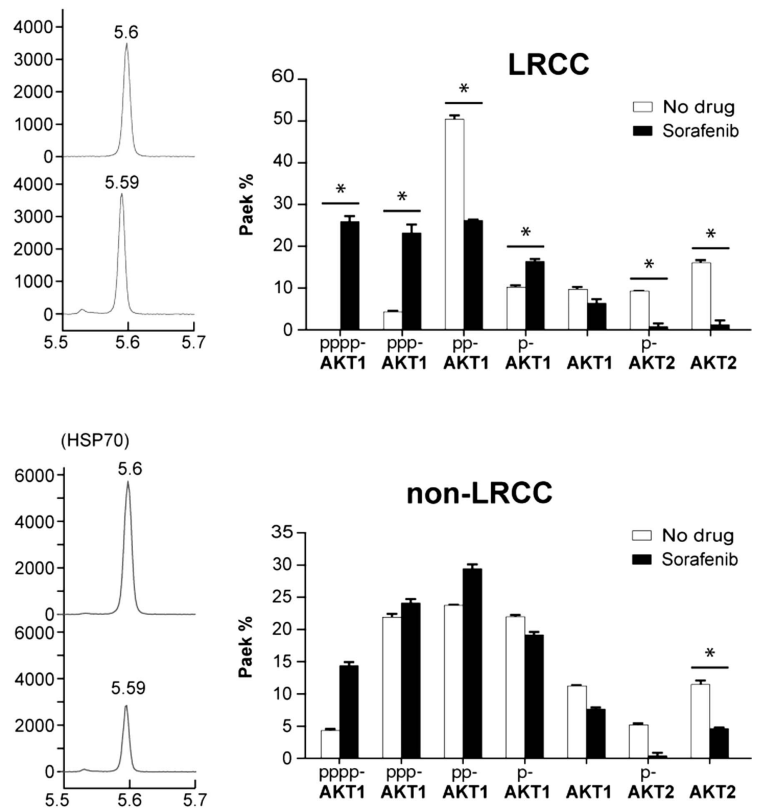

Figure 3 Extracellular-signal-regulated-kinases (ERK) and v-akt-murine-thymoma-viral-oncogene-homologue (AKT) are upregulated in label-retaining cancer cells (LRCC) after treatment with sorafenib. (A) After treatment with sorafenib ERK is upregulated in LRCC but not in non-LRCC (PLC/PRF/5 cells; $p=0.0046$ and $p=0.046$ ). (B) Activated AKT1 isoforms are upregulated in LRCC (of HuH-7 cells; $p=0.0020$ and $\mathrm{p}=0.0037) ;$ no statistically significant changes were observed in non-LRCC ( $\left.{ }^{*} \mathrm{p}<0.05\right)$.

Compared with non-LRCC, LRCC demonstrate improved viability, toxicity profiles and reduced apoptosis after treatment with sorafenib

To investigate potential mechanisms explaining the increased relative proportion of LRCC after treatment with sorafenib, we tested LRCC and non-LRCC after treatment with clinically relevant dosages of sorafenib for viability, toxicity and apoptosis. LRCC and non-LRCC were treated with sorafenib for eight cell cycles ( $\mathrm{n}=6$, Materials and methods section).

LRCC demonstrate improved viability over non-LRCC by $179 \%$ (figure 2D). The changes in viability were not uniform among all cell lines: PLC/PRF/5 4.26 \pm 1.58 , HuH-7 1.67 \pm 0.023 , SK-Hep-1 $2.10 \pm 0.080$ and combined $1.79 \pm 0.074$ (figure 2D). After treatment with sorafenib, almost twice as many LRCC are viable than non-LRCC. In comparison, there were no statistically significant inherent differences between LRCC and non-LRCC in terms of susceptibility to apoptosis or toxicity in the absence of sorafenib treatment (see online supplementary table S2). These results support our previous data suggesting that after treatment with sorafenib the relative proportion of LRCC is increased and that this phenomenon is accompanied by improved viability.

To further investigate potential mechanisms contributing to the survival advantage of LRCC over non-LRCC after treatment with sorafenib, we tested apoptosis and cytotoxicity. We tested apoptosis by measuring the activity of the effector caspases $3 / 7$. We detected a twofold decrease in the activities of caspases 3/7 
in LRCC compared with non-LRCC after treatment with sorafenib: PLC/PRF/5 -7.4 $\pm 0.3, \mathrm{HuH7}-4.8 \pm 0.07$, SK-Hep $-11.2 \pm 1.6$ and combined $-2.1 \pm 0.7$ fold decreased apoptosis. These data suggest that LRCC undergo less apoptosis than non-LRCC after treatment with sorafenib supporting our previous data (figure 2E).

In parallel, we measured toxicity by dead cells' protease release (Materials and methods section). LRCC sustained twofold less toxicity than non-LRCC after treatment with sorafenib. LRCC toxicity profile paralleled the apoptosis profile: PLC/PRF/5 -0.26 $\pm 0.85, \quad$ HuH7 $-5.98 \pm 1.16, \quad$ SK-Hep-1 $0.17 \pm 0.14$ and combined $2.02 \pm 0.67$ fold decreased toxicity after treatment with sorafenib compared with non-LRCC (figure 2F). These data support our previous results, and suggest that the increased relative proportion of LRCC over non-LRCC after treatment with sorafenib could potentially be explained by LRCC's relative resistance to apoptosis leading to improved LRCC viability and less cytotoxicity. Taken together these data suggest that LRCC are relatively resistant to sorafenib.

\section{Sorafenib treatment results in paradoxical activation of ERK and AKT in LRCC}

To further investigate the relative resistance of LRCC to sorafenib, we tested key sorafenib target proteins (STP) in LRCC, and non-LRCC before and after treatment with sorafenib. Sorafenib is a TKI with inhibitory effects on two main signal cascades: RTK-Ras-Raf-mitogen-activated protein kinase kinase (MEK)extracellular-signal-regulated kinases (ERK) and RTK(Ras)-PI3K-v-akt-murine-thymoma-viral-oncogene homologue (AKT)-mammalian target of rapamycin (see online supplementary data and table 1). Data reported by others demonstrated that the clinical effects of sorafenib are mediated via the inhibition of MEK, ERK and $\mathrm{AKT}^{3}{ }^{13}$ Therefore, we quantitatively tested total MEK1/ 2 and phosphorylated (activated) MEK (pS218/222, pT292 and pT386), total and phosphorylated ERK1/2, and AKT1/2.

Total and phosphorylated MEK1/2 are effectively suppressed by sorafenib in both LRCC and non-LRCC. We detected significant differences in ERK and AKT. In PLC/PRF/5, we detected higher levels of phosphorylated (activated) ERK in LRCC after treatment with sorafenib: ppERK1 $(\mathrm{p}=0.0045)$, ppERK2 $(\mathrm{p}=0.046)$, pERK2 $(\mathrm{p}=0.021)$ and a decrease in pERK1 $(p=0.025)$ (figure $3 \mathrm{~A}$, all measurements were normalised to house-keeping heat shock protein 70 ). In parallel, we detected statistically significant decrease in all ERK species in non-LRCC (figure 3A). In $\mathrm{HuH} 7$, we detected statistically significant increase in levels of the most powerful activated AKT1 isoforms (multiple-phosphorylated) in LRCC after treatment with sorafenib (ppppAKT1, $\mathrm{p}=0.0020$ and pppAKT1, $\mathrm{p}=0.0037$; figure 3B). The non-or-less-phosphorylated forms of AKT1 (ppAKT1 and AKT1) were suppressed while pAKT1 was upregulated $(p=0.018)$. In non-LRCC, there were no statistically different changes in levels of AKT1. In both LRCC and non-LRCC, AKT2 were suppressed effectively after treatment with sorafenib. Because we observed differences after treatment with sorafenib in ERK and AKT, we tested LRCC versus non-LRCC without sorafenib. ERK and AKT expression in LRCC was similar to non-LRCC or lower with one exception; ppAKT1 was expressed twice as high in LRCC than in non-LRCC (see online supplementary table S3). Our data suggest that in contrast to previous studies where ERK and AKT were suppressed after treatment with sorafenib in HCC cell lines, ${ }^{3}{ }^{13}$ here we show statistically significant elevation in the activated forms of ERK1/2 and AKT1 in LRCC. These data suggest that LRCC might resist some of the effects of sorafenib by modulating their response in terms of ERK and AKT. Because this phenomenon is not uniform across all cell lines, we propose that LRCC derived from different cell lines might apply different mechanisms to resist sorafenib treatment possibly by modulating different components of the MEK-ERK-AKT pathways. Moreover, because MEK-ERK-AKT expression is not different in untreated LRCC and non-LRCC, these data potentially suggest that it is possible that LRCC upregulate or alternatively decrease degradation of specific STP as a result or as a response of being exposed to sorafenib.

\section{STG are differentially expressed in LRCC and non-LRCC}

Sorafenib has potential downstream effects on multiple genes. Literature search resulted in proven effects on 60 genes herein, STG (table 1). To gain further understanding into the relative resistance of LRCC to sorafenib, we performed qRT-PCR SuperArray analysis on all cell lines tested (figures 4 and 5, online supplementary table S4): STG (60 genes), wingless-type MMTV-integration-site family (WNT) (84 genes), and stem cells associated genes (84 genes) in LRCC and non-LRCC after treatment with sorafenib.

Comparing LRCC versus non-LRCC after treatment with sorafenib, three STG demonstrated differential expression: BCL2L1 and FLT4 were upregulated 139 $\pm 4.3(\mathrm{p}=0.020)$ and $4.7 \pm 1.2(p=0.00066)$ fold, respectively (figure $4 A)$. Vimentin (VIM) was downregulated $160 \pm 17.0$ fold $(\mathrm{p}=0.019$, figure 4A). BCL2L1 can act as antiapoptotic factor. ${ }^{14}$ FLT4 encode for VEGF-C, involved in lymphangiogenesis, and interact with src-homology-2-domain-containing- transforming-protein-1 and basic-helix-loop-helix-transcription-factor-SCL (SCL, see online supplementary data) to suppress apoptosis and induce cell proliferation via ERK. VIM encodes for Vimentin, an intermediate filament involved in cytoskeleton stabilisation and epithelialmesenchymal transition.

Testing STG in LRCC before and after treatment with sorafenib demonstrated a dramatic upregulation of the CSC marker aldehyde dehydrogenase 1 family (ALDH1)A1 (figure 4B, $20680 \pm 10480$ fold, $p=0.0093$ ), and downregulation of the pro-apoptotic gene EIF2S1 $(373.8 \pm 185.0$ fold, $p=0.049)$. In contrast to previous reports, here we show that sorafenib has diametrically opposite effects on BCL2L1, ${ }^{14}$ FLT4, ${ }^{3}$ ALDH $1 \mathrm{~A} 1^{15}$ and EIF2S $1^{16}$ in LRCC. These results are consistent with the observation that LRCC exhibit reduced apoptosis and demonstrated improved viability profiles when compared with non-LRCC and the increase in relative proportion of LRCC after treatment with sorafenib.

The Wnt pathway regulates stem cells differentiation and proliferation. Previously, we demonstrated that LRCC are stem-like TIC. ${ }^{10}{ }^{11}$ Therefore, we investigated Wnt pathway genes in LRCC versus non-LRCC after treatment with sorafenib (figure 5A). We found that the Wnt agonist WNT9A and the Wnt target gene Cyclin D2 (CCND2) are upregulated in LRCC $(3.2 \pm 0.5$ fold, $p=0.016$ and $5.4 \pm 1.9$ fold, $p=0.015) .{ }^{17} \mathrm{We}$ tested LRCC alone before and after treatment with sorafenib (figure 5B). We found that the transcription coactivator pygopus homologue-1 (Pygo1) was upregulated $(2.9 \pm 0.5$ fold, $\mathrm{p}=0.041)$ and that WNT16 is downregulated $(6.0 \pm 1.6$ fold, $\mathrm{p}=0.031)$. Pygo1 is a nuclear protein associated with $\beta$-catenin that is required for TCF-mediated transcription. ${ }^{18}$ WNT16 regulates cellular senescence via p53 activity. ${ }^{19}$ Upregulation of the Wnt pathway and downregulation of WNT16 can result in increased cell proliferation, self-renewal and reduced cell senescence. ${ }^{17-19}$ These results are consistent with the observation that LRCC demonstrated improved viability and toxicity profiles and 
Figure 4 Sorafenib target genes (STGs) expression in label-retaining cancer cells (LRCC) versus non-LRCC (all three hepatocellular carcinoma cell lines tested). (A) Compared with non-LRCC, sorafenib treatment of LRCC resulted in opposite effects by upregulation of BCL2L1 and

Fms-related tyrosine kinase4 and downregulation of VIM. (B) STGs expression in LRCC before and after treatment with sorafenib $\left({ }^{*} p<0.05\right)$.

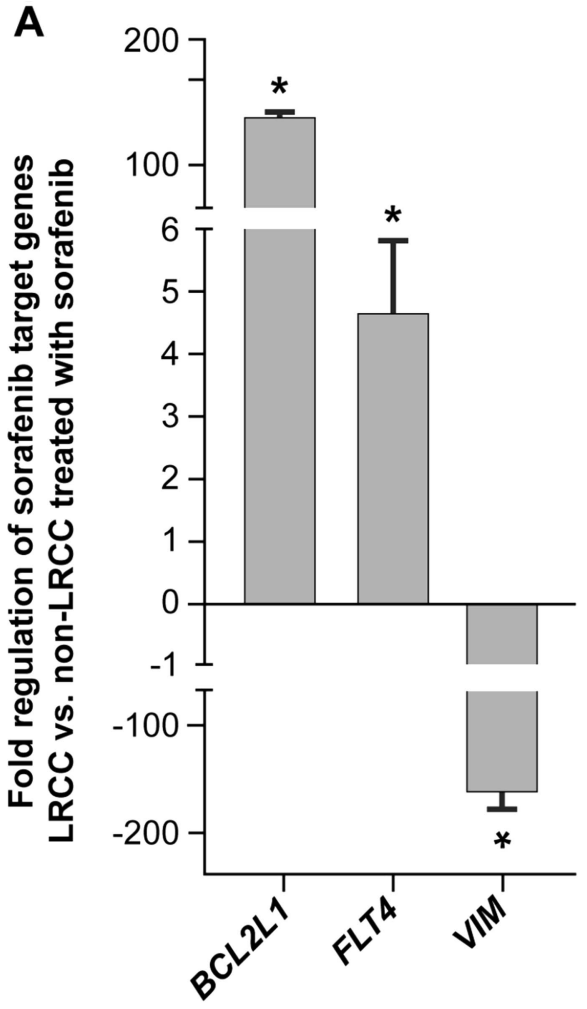

reduced apoptosis than non-LRCC after treatment with sorafenib; in addition, there is a corresponding sorafenib-associated increase in the relative proportion of LRCC after treatment with sorafenib.

Similarly, we tested the expression of stem cells associated genes in LRCC versus non-LRCC after treatment with sorafenib. We found that five genes were downregulated in LRCC compared with non-LRCC (figure 5C): stem cell differentiation gene ISL-LIM-homeobox-1 (ISL1, $-328 \pm 313$ fold, $\mathrm{p}=0.0066$ ) promoting organogenesis; fibroblast-growth-factor-3 (FGF3, -7.1 2.3 fold, $\mathrm{p}=0.01$ ) promoting cell proliferation and survival; cell adhesion-related gap-junction-protein-B1 (GJB1, $-6.5 \pm 2.3$ fold, $\mathrm{p}=0.0011$ ); MYST-histone-acetyltransferase-2 (MYST2, -114.1 \pm 26.8 folds, $\mathrm{p}=0.00001$ ) required for formation of blood vessels, mesenchyme and somites; and the Notch signalling inhibitor Numb-homologue (NUMB, -101.2 \pm 24.1 fold, $p=0.000008$ ) regulating self-renewal and asymmetric cell division. Finally, we tested LRCC before and after treatment with sorafenib. We found that 12 genes were downregulated (figure 5D): cell cycle arrest gene retinoblastoma 1 (RB1, $-5.9 \pm 1.0$ folds, $\mathrm{p}=0.000009)$; cell adhesion-related genes Rho GTPase cell-division-cycle-42 (Cdc42, $-3.7 \pm 0.7, p=0.00012)$ involved in extracellular matrix remodelling and cell polarity, GJB1 $(-78.5 \pm 21.57$ fold, $\mathrm{p}=0.029)$, catenin-A1 (CTNNA1, -46.1 \pm 12.31 fold, $p=9 \mathrm{E}-5)$, cardiac-muscle- $\alpha$-actin-1 (ACTC1, $-29.9 \pm 11.30$ fold, $p=0.034$ ) and aggrecan (ACAN, $-51.3 \pm 19.4$ fold, $\mathrm{p}=0.013$ ); liver stem cell differentiation gene forkhead-box-A2 (FOXA2, -95.1 \pm 12.29 fold, $p=0.0075)$, neural stem cell self-renewal and differentiation genes sex-determining-region-Y-box-1 (SOX1, $-95.5 \pm 5.09$ fold, $\mathrm{p}=0.036)$ and $\beta$-tubulin-3 (TUBB3, $-3.0 \pm 0.6$ fold, $\mathrm{p}=0.0026$ ), Notch-homologue-2 (Notch2, $-128.6 \pm 36.23$ fold, $\mathrm{p}=0.00039$ ), Wnt pathway repressor $\beta$-transducin-repeat-containing (BTRC, $-9.5 \pm 5.9$ fold, $\mathrm{p}=0.017)$ and FGF3 $(-23.0 \pm 3.7$ fold, $\mathrm{p}=0.018)$.

Taken together, we show that sorafenib treated LRCC upregulate cell proliferation genes, the CSC marker gene ALDH1,
Wnt genes and cell survival genes, and downregulate cell cycle arrest genes, apoptosis genes, cell adhesion genes and stem cell differentiation genes. To integrate the protein and the gene profiling results, we used the IPA software to generate a molecular pathway map for LRCC after treatment with sorafenib (figure 6).

\section{DISCUSSION}

We undertook these studies to test the hypothesis whether liver cancer derived CSC (ie, LRCC) are more resistant to therapy than the non-CSC counterpart (ie, non-LRCC). Here we show that the relative proportion of LRCC significantly increased after treatment with sorafenib. This CSC (LRCC) subpopulation increase was accompanied by less apoptosis, and improved viability and toxicity profiles than non-LRCC, and associated with non-uniform AKT and ERK activation. Additionally, LRCC treated by sorafenib upregulate the CSC marker ALDH1, Wnt, cell survival and proliferation genes, and downregulate apoptosis, cell cycle arrest, cell adhesion and differentiation genes. Our data suggest that LRCC are relatively resistant to sorafenib. This is the first report suggesting that HCC derived CSC are resistant to targeted therapy. Given the ability of LRCC to form tumours with only 10 cells, ${ }^{11}$ we propose a role for LRCC in HCC recurrence.

Several investigators hypothesised that LRCC and other CSC are relatively quiescent and, therefore, sorafenib induced cell death will not manifest unless cells are exposed over time. ${ }^{5} 9$ Here, we exposed LRCC and non-LRCC to clinically relevant sorafenib concentrations over prolonged period of time (eight cell cycles). In parallel, we demonstrated that this dose, over the same period of time, is very effective against all cell lines tested (bulk cells). Moreover, we previously demonstrated that HCC derived LRCC undergo active cell division and are generated as a result of asymmetric cell division and not cell quiescence. ${ }^{10} 11$ Thus, we suggest that the increase in the relative proportion of 
A

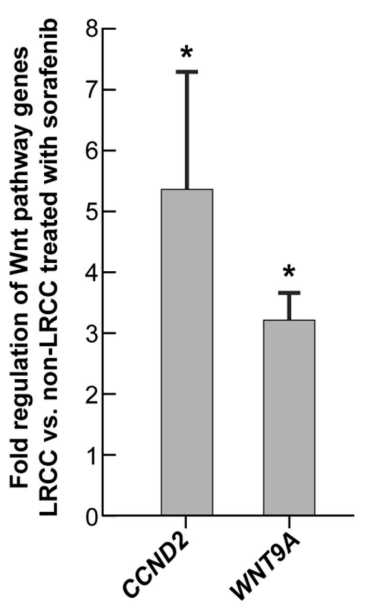

B

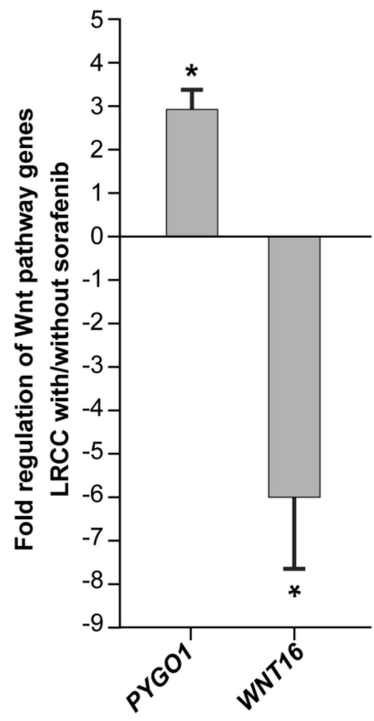

C

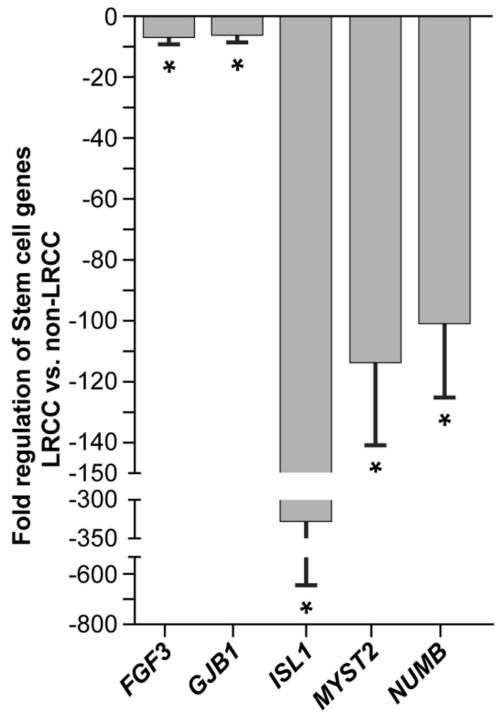

D

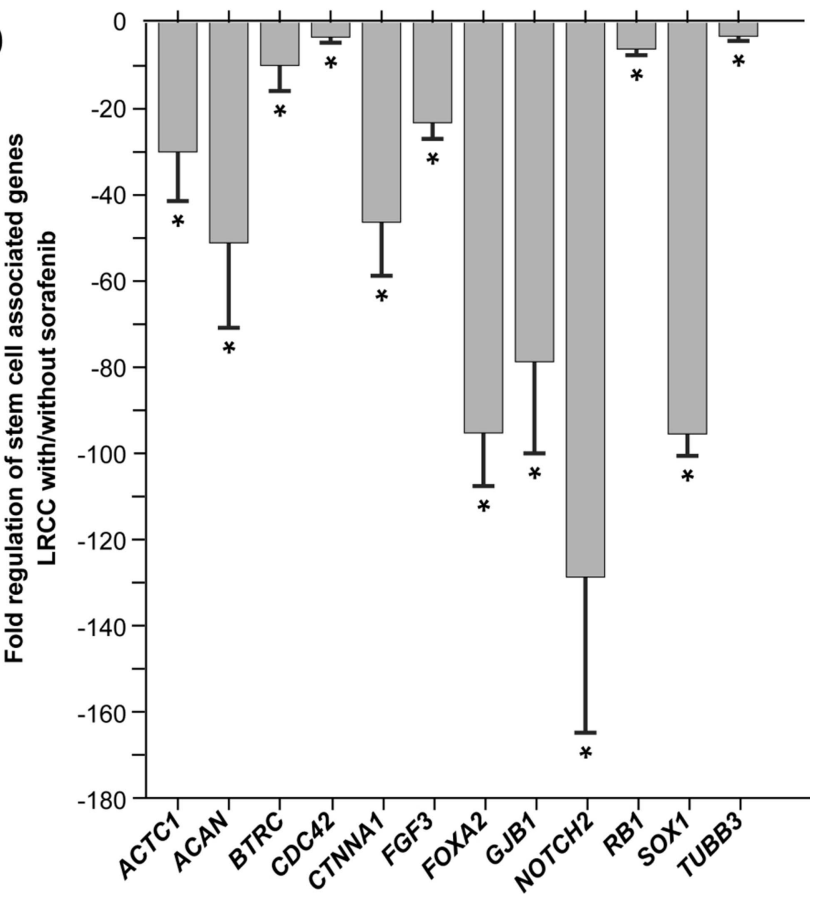

Figure 5 Wnt pathway and stem cells associated genes expression (all three hepatocellular carcinoma cell lines tested). (A) Wnt pathway genes' expression in label-retaining cancer cells (LRCC) versus non-LRCC after treatment with sorafenib and (B) in LRCC with/without sorafenib. (C) Stem cells associated genes' expression in LRCC versus non-LRCC after treatment with sorafenib and (D) in LRCC with/without sorafenib ( $\left.{ }^{*} p<0.05\right)$.

LRCC after treatment with sorafenib is a manifestation of innate resistance to xenobiotic-stressors like sorafenib inherent to LRCC.

In agreement with the increased relative proportion of LRCC, we observed increased viability of LRCC versus non-LRCC after treatment with sorafenib. However, while apoptosis and toxicity profiles validated these results, in Sk-Hep-1 there was little change in apoptosis. Interestingly, we detected the largest increase in the relative proportion of LRCC in Sk-Hep1. How can we explain such a dramatic increase (over 16-fold, figure $2 \mathrm{C}$ ) in the relative proportion of LRCC with little difference in the apoptosis profile of Sk-Hep-1 derived LRCC (figure 2E)? It is possible that various LRCC derived from different cell lines, original locations (primary vs metastatic sites) and stages will have different mechanisms to avoid the full effect of sorafenib. Sk-Hep-1 was derived from ascites and from a poorly differentiated tumour while $\mathrm{HuH}-7$ and PLC/PRF/5 were derived from a well differentiated primary liver HCC. Another possible explanation could be based on the fact that in stem cells, caspase 3 can function both as inducer of differentiation/growth and apoptosis. ${ }^{20}$ Thus, it is possible to detect higher levels of caspase 3 without concomitant increase in apoptosis in stem cells. In fact, one would expect higher proliferation than expected potentially explaining the dramatic increase in the relative proportion of LRCC without significant changes in apoptosis, as seen here. Finally, it has been reported that sorafenib induces apoptosis in HCC cell lines both in a caspase-dependent and caspase-independent fashion. ${ }^{21}$ Thus, one cannot conclude 


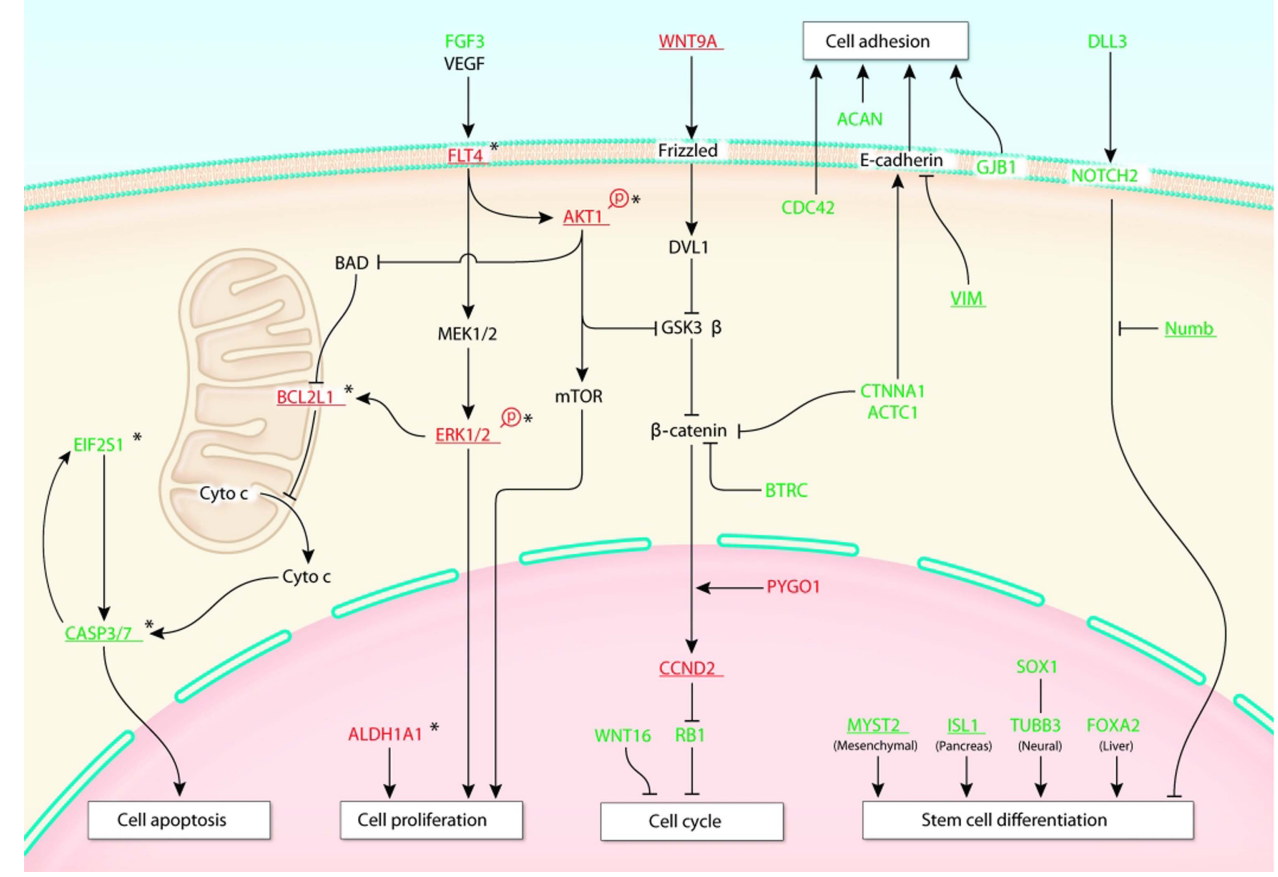

Figure 6 A pathway map for sorafenib treated label-retaining cancer cells (LRCC). Ingenuity Pathway Analysis of protein and gene expression data from all three hepatocellular carcinoma cell lines. Red/green: upregulation and downregulation, respectively; underlines: LRCC versus non-LRCC; asterisk: upregulation/downregulation in an opposite direction to previous reports on whole cell populations. The map suggests a potential proliferative, more metastatic and less differentiated with less apoptosis expression profile for sorafenib treated LRCC.

definitively that the relative resistance of LRCC to sorafenib is based on their pure ability to evade caspase-dependent apoptosis. It is likely to be a more complex, multi-faceted and redundant mechanism. Our results indicate that LRCC exhibit survival advantage over non-LRCC after treatment with sorafenib resulting in a significant increase in the relative proportion of LRCC among all HCC cell lines tested. Since LRCC can generate tumours with as little as 10 cells $^{10}{ }^{11}$ we hypothesise that this phenomenon can potentially contribute to liver cancer recurrence after treatment with sorafenib.

To determine whether the relative resistance of LRCC to sorafenib was accompanied by changes in STP, we tested total and activated (phosphorylated) MEK1/2, ERK1/2 and AKT1/2. Our data suggest that in contrast to previous studies where ERK and AKT were suppressed in HCC cell lines after treatment with sorafenib, ${ }^{3} 13$ here we show statistically significant elevation in the activated forms of ERK and AKT in LRCC but not in non-LRCC. Other authors reported similar findings further validating our results. Kuen-Feng Chen et al ${ }^{22}$ reported that resistance to sorafenib in HuH-7 and Sk-Hep-1 is mediated via activation of AKT. The same authors found that MEK1/2 is downregulated in sorafenib resistant and sensitive cells, as shown in this report. In contrast, they found that sorafenib resistant HCC cells downregulate ERK while we found that activated ERK is upregulated in LRCC after treatment with sorafenib. The difference between these two reports can be based on the fact that Kuen-Feng Chen et al analysed whole HCC cell lines and we analysed HCC derived LRCC which are putative HCC CSC. ${ }^{11}$ These data suggest that LRCC might resist some of the effects of sorafenib by different responses in terms of ERK and AKT. We propose that LRCC derived from different cell lines may apply different mechanisms to cope with the biological stress induced by sorafenib treatment with variable involvement of STP.

Finally, to gain further insight into the relative resistance of LRCC to sorafenib, we tested STG. Previously, we reported that
LRCC upregulate Wnt pathway genes and highly express pluripotency genes. ${ }^{11}$ Thus, we further tested the effects of sorafenib on Wnt pathway and stem cells associated genes in LRCC and non-LRCC after treatment with sorafenib. We showed that sorafenib has opposing effects on four key STG (ALDH1A1, ${ }^{15}$ BCL2L1, ${ }^{14}{ }^{21}$ EIF2S1 $^{16}$ and FLT4 ${ }^{3}$ ) in LRCC compared with non-LRCC, and previously published data (table 1 ). These data suggest that LRCC respond differently to sorafenib treatment. Upregulation of FLT4 promotes cell proliferation and inhibits apoptosis through activations of the MAP-kinase, AKT and BCL2L1. ${ }^{3}$ Upregulated BCL2L1 and downregulated EIF2S1 can inhibit cytochrome $\mathrm{C}$ release leading to inhibition of apoptosis. ${ }^{161}$ These findings further support our data.

Testing stem cells associated genes in LRCC versus non-LRCC after treatment with sorafenib, we found that LRCC downregulate cell adhesion genes (ACAN, CDC42, CTNNA1 and GJB1), stem cell differentiation genes (FOXA2, ISL1, MYST2, Numb and TUBB3) and upregulate the CSC marker ALDH1A1. Downregulation of cell adhesion genes is consistent with decreased cell-matrix and cell-cell adhesion suggesting a more aggressive or metastatic phenotype. Downregulation of FOXA2, ISL1, MYST2, Numb and TUBB3 suggest that sorafenib treated LRCC may be less differentiated. The dramatic upregulation of ALDH1A1 suggests that sorafenib treated LRCC have high metabolic and proliferative activities. Overall, these data suggest that LRCC have a metastatic-like less differentiated gene expression profile after treatment with sorafenib.

Compared with non-LRCC, we detected statistically significant changes in several Wnt pathway genes in LRCC: upregulation of WNT9A and PYGO1, ${ }^{18}$ and downregulation of BTRC, WNT1 $6^{19}$ and CTNNA1. These changes can activate CCND2 and inhibit the cell cycle arrest gene RB1, leading to cell cycle progression and enhanced proliferation. Downregulation of the Notch inhibitor Numb suggests that sorafenib treated LRCC could maintain stem cell self-renewal capacity via Notch 
signalling. Of note, we reported recently that Wnt is a major positive regulator of LRCC undergoing self-renewal asymmetric cell division (Xin et al unpublished data). However, not all gene expression changes follow these trends and some are inconsistent with these results (downregulation of Notch2, Notch ligand DLL3 and FGF3). To integrate these findings, we used the Ingenuity software to generate a molecular pathway map of sorafenib treated LRCC (figure 6).

In conclusion, here we show that the recently described stemlike cancer cells, that is, LRCC are relatively resistant to sorafenib. After treatment with sorafenib, the relative proportion of LRCC increased dramatically compared with non-LRCC. These results were further supported by better viability, apoptosis and toxicity profiles, and STP and STG profiles in LRCC than non-LRCC. These data suggest a potential role for LRCC in HCC progression after treatment with sorafenib. Further elucidation of this phenomenon may provide novel strategies to target HCC and/or enhance the efficacy of sorafenib.

\section{Author affiliations}

'Surgery Branch, Center for Cancer Research, National Cancer Institute, National Institutes of Health, Bethesda, Maryland, USA

${ }^{2}$ Collaborative Protein Technology Resource, Center for Cancer Research, National Cancer Institute, National Institutes of Health, Bethesda, Maryland, USA

${ }^{3}$ Department of Surgery, Division of Surgical Oncology, Walter Reed National Military Medical Center, Bethesda, Maryland, USA

${ }^{4}$ Department of Surgery, Uniformed Services University of the Health Sciences, Bethesda, Maryland, USA

${ }^{5}$ Laboratory for Experimental Carcinogenesis, Center for Cancer Research, National

Cancer Institute, National Institutes of Health, Bethesda, Maryland, USA

${ }^{6}$ Bon Secours Cancer Institute, Richmond, Virginia, USA

Acknowledgements This study was supported by the intramural grant NC intramural grant 1ZIABC011005 provided by the NIH/National Cancer Institute. We thank Madeleine R Heldman for her help in NanoPro data quantification and figure preparation.

Contributors Conception and design: HWX, SST, IA; Administrative support: SR, UR, SST, IA; Collection of data: HWX, CMA, GWW, TCM, JQC, AJA, SR, DB, MAH; Data analysis and interpretation: HWX, GWW, JQC, UR, SST, IA; Manuscript writing: HWX, CMA, DMH, JEM, TK, RCL, PKG, AS, UR, SST, IA; Final approval of manuscript: UR, SST, IA.

Funding The NIH/National Cancer Institute, NCI intramural grant 1ZIABC011005.

Competing interests None.

Provenance and peer review Not commissioned; externally peer reviewed.

\section{REFERENCES}

1 Jemal A, Siegel R, Ward E, et al. Cancer statistics, 2007. CA Cancer J Clin 2007:57:43-66.
2 Llovet JM, Ricci S, Mazzaferro V, et al. Sorafenib in advanced hepatocellular carcinoma. N Engl J Med 2008;359:378-90

3 Wilhelm S, Carter C, Lynch M, et al. Discovery and development of sorafenib: a multikinase inhibitor for treating cancer. Nat Rev Drug Discov 2006;5:835-44.

4 Lapidot T, Sirard C, Vormoor J, et al. A cell initiating human acute myeloid leukaemia after transplantation into SCID mice. Nature 1994;367:645-8.

5 Visvader JE, Lindeman GJ. Cancer stem cells in solid tumours: accumulating evidence and unresolved questions. Nat Rev 2008;8:755-68.

6 Capper D, Gaiser T, Hartmann C, et al. Stem-cell-like glioma cells are resistant to TRAIL/Apo2L and exhibit down-regulation of caspase-8 by promoter methylation. Acta Neuropathol 2009;117:445-56.

7 Corbin AS, Agarwal A, Loriaux M, et al. Human chronic myeloid leukemia stem cells are insensitive to imatinib despite inhibition of BCR-ABL activity. J Clin Invest 2011:121:396-409.

8 Smith GH. Label-retaining epithelial cells in mouse mammary gland divide asymmetrically and retain their template DNA strands. Development 2005;132:681-7.

9 Kuwahara R, Kofman AV, Landis CS, et al. The hepatic stem cell niche: identification by label-retaining cell assay. Hepatology 2008;47:1994-2002.

10 Hari D, Xin HW, Jaiswal K, et al. Isolation of live label-retaining cells and cells undergoing asymmetric cell division via nonrandom chromosomal cosegregation from human cancers. Stem Cells Dev 2011;20:1649-58.

11 Xin HW, Hari DM, Mullinax JE, et al. Tumor initiating label-retaining-cancer-cells in human gastrointestinal cancers undergo asymmetric cell division. Stem Cells 2012:30:591-8

12 Keating GM, Santoro A. Sorafenib: a review of its use in advanced hepatocellular carcinoma. Drugs 2009;69:223-40.

13 Gedaly R, Angulo P, Hundley J, et al. Pl-103 and sorafenib inhibit hepatocellular carcinoma cell proliferation by blocking Ras/Raf/MAPK and PI3K/AKT/mTOR pathways. Anticancer Res 2010:30:4951-8.

14 Rosato RR, Almenara JA, Coe S, et al. The multikinase inhibitor sorafenib potentiates TRAIL lethality in human leukemia cells in association with Mcl-1 and cFLIPL down-regulation. Cancer Res 2007;67:9490-500.

15 Rausch V, Liu L, Kallifatidis G, et al. Synergistic activity of sorafenib and sulforaphane abolishes pancreatic cancer stem cell characteristics. Cancer Res 2010;70:5004-13.

16 Park MA, Zhang G, Martin AP, et al. Vorinostat and sorafenib increase ER stress, autophagy and apoptosis via ceramide-dependent CD95 and PERK activation. Cancer Biol Ther 2008:7:1648-62.

17 Jane EP, Premkumar DR, Pollack IF. Coadministration of sorafenib with rottlerin potently inhibits cell proliferation and migration in human malignant glioma cells. J Pharmacol Exp Ther 2006;319:1070-80.

18 Thompson B, Townsley F, Rosin-Arbesfeld $\mathrm{R}$, et al. A new nuclear component of the Wnt signalling pathway. Nat Cell Biol 2002;4:367-73.

19 Binet R, Ythier D, Robles Al, et al. WNT16B is a new marker of cellular senescence that regulates p53 activity and the phosphoinositide 3-kinase/AKT pathway. Cancer Res 2009;69:9183-91.

20 Abdul-Ghani M, Megeney LA. Rehabilitation of a contract killer: caspase-3 directs stem cell differentiation. Cell Stem Cell 2008;2:515-16.

21 Panka DJ, Wang W, Atkins MB, et al. The Raf inhibitor BAY 43-9006 (Sorafenib) induces caspase-independent apoptosis in melanoma cells. Cancer Res 2006:66:1611-19.

22 Chen KF, Chen HL, Tai WT, et al. Activation of phosphatidylinositol 3-kinase/Akt signaling pathway mediates acquired resistance to sorafenib in hepatocellular carcinoma cells. J Pharmacol Exp Ther 2011;337:155-61. 